\title{
Protein-based prognostic biomarkers in lung cancer: Promise or pitfall?
}

\author{
Chuong D. Hoang, MD \\ From the Section of Thoracic Surgery, National Institutes of Health-National Cancer Institute, Bethesda, Md. \\ Disclosures: Author has nothing to disclose with regard to commercial support. \\ Received for publication April 9, 2017; accepted for publication April 12, 2017; available ahead of print May 11, \\ 2017. \\ Address for reprints: Chuong D. Hoang, MD, Section of Thoracic Surgery, National Institutes of Health-National \\ Cancer Institute, CCR and The Clinical Center, 10 Center Dr, Room 4-3940, Mail code 1201, Bethesda, MD \\ 20892 (E-mail: chuong.hoang@nih.gov). \\ J Thorac Cardiovasc Surg 2017;154:1418-9 \\ 0022-5223/\$0.00 \\ Published by Elsevier Inc. on behalf of The American Association for Thoracic Surgery \\ http://dx.doi.org/10.1016/j.jtcvs.2017.04.032
}

Non-small cell lung cancer (NSCLC) is driven by a highly complex, yet orchestrated set of gene programs, the precise molecular mechanisms of which continue to defy our complete understanding. ${ }^{1}$ Ultimately, the biologic heterogeneity of NSCLC obscures our ability to predict its behavior accurately. This is implied by iterative updates of the lung cancer TNM staging system, because it is well recognized that prognosis within each currently defined stage may vary significantly across individual patients. ${ }^{2}$ Many strategies of integrating molecular prognostication into routine pathologic diagnosis to improve the management, treatment, and survival of patients with NSCLC have been attempted, but without subsequent successful clinical adoption of a new paradigm. Molecular prognostic markers can be proteins, messenger RNA, DNA, microRNA, metabolites, or combinations of any of these classes of biologic molecules. The most extensively studied markers to date in NSCLC are proteins, typically assessed by immunohistochemistry.

In this issue of the Journal, Shimizu and colleagues ${ }^{3}$ present their retrospective institutional experience with a large cohort of patients with lung adenocarcinoma for whom they used immunohistochemical staining of tumors to evaluate expression of stathmin 1 (STMN1), a protein observed in other solid tumors that participates in regulation of cellular proliferation. They observed an overall prevalence of detectable STMN1 in 54\% of lung adenocarcinomas. Among clinicopathologic associations, they identified novel links of high STMN1 protein expression with high microvessel density and positive lymphovascular invasion. Stringent statistical modeling showed that STMN1 protein expression was an independent negative prognostic factor. Most interestingly, STMN1 expression was found to be an independent negative prognostic factor for overall survival in all patients who underwent surgical resection and had recurrence. Even within the stage I group, further substratification of outcome was possible on the basis of STMN1 status.

Despite these promising features of STMN1 for adenocarcinoma, several hundreds of reports $(>500)$ during the

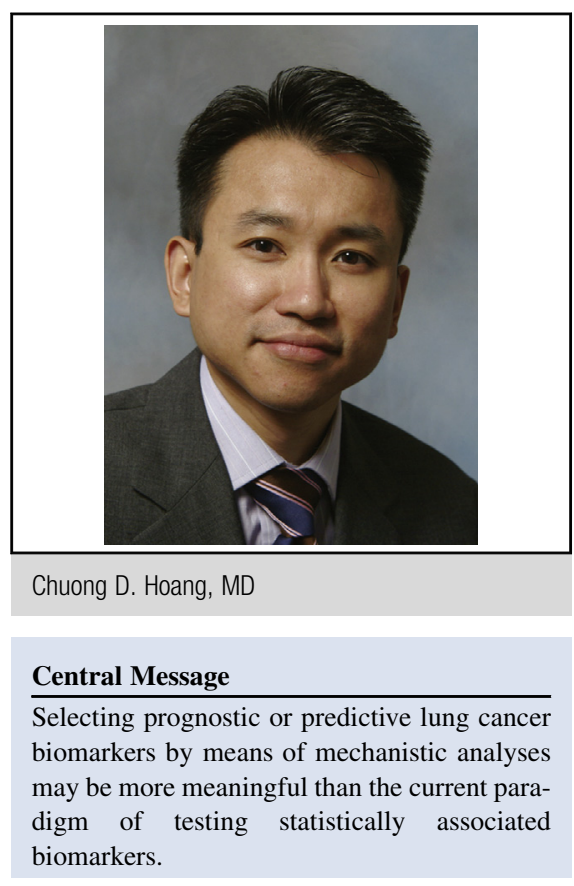

See Article page 1406. past 30 years have neither identified nor validated sufficiently a single protein lung cancer biomarker for clinical use. ${ }^{4}$ In fact, immunohistochemistry-based assays have often given rise to inconsistent or contradictory results. Recently, a high-profile cautionary example of this problem is provided by the failure of excision repair crosscomplementation group (ERCC1) protein to predict outcome and cisplatin response in patients with NSCLC. ${ }^{5}$ A cross-validation study performed within an international meta-analysis effort (Lung Adjuvant Cisplatin Evaluation) could not establish ERCC1 as a lung cancer biomarker, because the commercially available antibodies failed to detect the biologically relevant protein isoform. Additionally, in a broad sense, immunohistochemical techniques are generally vulnerable to lack of standardization in multiple factors involved in specimen processing (where to sample within the tumor mass, how many tissue slides to receive, etc), including the source and quality of the antibodies, the histologic staining protocol, diverse scoring algorithms used to determine positive versus negative expression status, qualitative interpretation of staining intensity, arbitrary cutoffs for dichotomizing scores, ${ }^{6}$ and the statistical methods used to analyze the data. These 
contrary factors are formidable barriers to overcome in the elusive process of implementing clinically useful immunohistochemistry-based biomarkers. It will be interesting to see any future validation results of STMN1 performance in an external cohort of patients with lung cancer and to ascertain reproducibility.

\section{References}

1. Chen Z, Fillmore CM, Hammerman PS, Kim CF, Wong KK. Non-small-cell lung cancers: a heterogeneous set of diseases. Nat Rev Cancer. 2014;14:535-46.

2. Detterbeck FC, Chansky K, Groome P, Bolejack V, Crowley J, Shemanski L, et al. IASLC Staging and Prognostic Factors Committee, Advisory Boards, and Participating Institutions. The IASLC Lung Cancer Staging Project: methodology and validation used in the development of proposals for revision of the stage classification of NSCLC in the forthcoming (eighth) edition of the TNM Classification of Lung Cancer. $J$ Thorac Oncol. 2016;11:1433-46.

3. Shimizu K, Ohtaki Y, Altan B, Yokobori T, Nagashima T, Arai M, et al. Prognostic impact of stathmin 1 expression in patients with lung adenocarcinoma. J Thorac Cardiovasc Surg. 2017:154:1406-17.

4. Zhu CQ, Shih W, Ling CH, Tsao MS. Immunohistochemical markers of prognosis in non-small cell lung cancer: a review and proposal for a multiphase approach to marker evaluation. J Clin Pathol. 2006;59: 790-800.

5. Friboulet L, Olaussen KA, Pignon JP, Shepherd FA, Tsao MS, Graziano S, et al. ERCC1 isoform expression and DNA repair in non-small-cell lung cancer. $N$ Engl J Med. 2013;368:1101-10.

6. Altman DG, Lausen B, Sauerbrei W, Schumacher M. Dangers of using "optimal" cutpoints in the evaluation of prognostic factors. J Natl Cancer Inst. 1994;86: $829-35$. 